\title{
Enterprise Resource Planning (ERP) Systems: Design, Trends and Deployment
}

\author{
Dania Mahmoud Bahssas \\ Department of Information Systems \\ Faculty of Computing \& Information Technology \\ King Abdulaziz University, Jeddah, Saudi Arabia \\ Email: dbahssas@hotmail.com \\ Dr. Adnan Mustafa AlBar \\ Department of Information Systems \\ Faculty of Computing \& Information Technology \\ King Abdulaziz University, Jeddah, Saudi Arabia \\ Email:ambar@kau.edu.sa \\ Md. Rakibul Hoque* \\ Department of Management Information Systems \\ Faculty of Business Studies \\ University of Dhaka, Dhaka-1000, Bangladesh \\ Email:rakibul@du.ac.bd
}

\begin{abstract}
The Enterprise Resource Planning (ERP) system is a famous widespread solution in business organization which is used and verified the integration and automation of the processes, performance improvements, and cost reduction. However, new innovations in technology trends that forced ERP designers to go with the new development. Therefore, new ERP system designs were done to satisfy companies and/or customers by evolving new ERP business models. Moreover, one of the biggest challenges for ERP is to keep speed with a manufacturing sector that has been rapidly moving from product-centric to customer-centric focus. This change required most ERP vendors to add a variety of functions and modules on their core systems, while the basic design of most ERP systems remained product-centric. In this paper, we discuss some of the promising new ERP evolutionary design and models that could affect the future of ERP systems and open new market for next generation customers.
\end{abstract}

Key words: Enterprise Resource Planning (ERP), ERP Design, ERP Architecture.

\section{Introduction}

Business environment is changing over the years, this pace of change continues to accelerate and companies all over the world seek to response to new customer needs and reach higher market opportunities. ${ }^{1}$ Successful companies today recognized the need of integrated systems that can improve their quality, customer satisfaction, performance, and profit. Organizations and companies can make this vision

*Corresponding author: Cell:+8801912928171.

Email: rakibul@du.ac.bd 
possible through Enterprise resource planning (ERP) systems. Enterprise resource planning (ERP) is business management software that is designed to integrate data sources and processes of an entire organization into a combined system. ${ }^{2}$ It gives organizations and companies an incorporated real-time view of its core business processes such as production, planning, manufacturing, inventory management, and development.

ERP software is super multi-module application software that integrates activities across functional departments such as production planning, purchasing, inventory control, product distribution, and order tracking. ${ }^{3}$ In addition, it allows automation and integration of business process by enabling data and information sharing to reach best practices in managing business process. ERP have different modules that run variety business activities such as accounting, finance, supply chain, human resources, customer information and many others.

\section{History of ERP}

ERP systems were used since 1960's and at the beginning, companies wanted a solution to control their inventory control systems by using Inventory control packages (IC). Then, in1970's Material Requirement Planning (MRP) Systems where developed for products planning followed by Manufacturing Resource Planning (MRP II) Systems developed in late 1980's to emphasis optimized manufacturing processes to form a character based ERP. ${ }^{4}$ In 1990, based on MRP and MRP II, ERP system where developed as Client/Server ERP to integrate business processes such as manufacturing, distributions, accounting, finance, human resources, , inventory management, and project management. ${ }^{5}$

In small words, between 1990 and 1999, ERP concept was based on materials planning, old entry, distribution, accounting, and shop floor control. In 2000, ERP vendor implement ERP system by adding modules and functions to have extended ERP (ERP II) system focusing on integration, transformation, and collaboration in the business value chain. ${ }^{6}$ New concept of ERP was proposed in 2001 to be internet based environment. In 2004, Services Oriented Architecture (SOA) becomes a standard that ERP vendors work towards. SOA software architecture allows different systems to communicate with each other. ${ }^{7}$ In 2005 , Industry consolidation occurs between companies such as oracle, Microsoft, Infor, and Sage. Extended ERP, between 2000 and 2005, was based on scheduling, forecasting, capacity planning, warehousing, and logistics. Furthermore, as business entered 21 st century the idea of extended ERP converted to be more sophisticated concept that includes all functions that was outside ERP such as knowledge management, project management, workflow management, human resource Management, customer relationship management, and integrated financials. ${ }^{8}$

\section{ERP Market}

In the early sixties, at the beginning SAP capitalized on innovations in client-server computing to be the leader in the ERP market. Later, database standardization by Oracle and Microsoft had a great impact on enterprise modules integration such as CRM. In addition, the beginning of web based software (SaaS) made a decrease in ERP system deploy cost and made ERP system to become the latest enterprise application to be available as a cloud. SaaS simplified the adoption of ERP and the extensibility of existing deployments. ${ }^{9}$

ERP Market is dominated by large vendors including SAP, Oracle, and Microsoft. Many ERP vendors had a strong presence among small to mid-sized enterprises market such as Sage, SAP, Oracle, Microsoft, Dynamics, NetSuite, Epicor, UNIT4 Business Software, and Openbravo. ERP market vendor landscape where based on business size categories which are: vendors serving large businesses such as Oracle and SAP that provide ERP software for multilanguage, multi-facility, billion-dollar enterprises, vendors serving mid-sized businesses such as Infor, Sage, and Epicor, and vendors serving small businesses where Epicor are the front runners in this segment. ${ }^{10}$

\section{Traditional ERP Design}

As old technology trends supported ERP design, architecture, and framework, therefore, Traditional ERP designs were implemented and made a conceptual framework for next-generation and future ERP systems. This section explains various ERP architecture design, technology used, issues, and challenges in each architecture design.

\subsection{Two-tier (client/server) architecture}

ERP architecture design was based on client/Server processing which means that processed work will be 
divided between two computers Client and Server. Client is the presentation logic for users while Server is the processing and storage logic. ${ }^{11}$ This design was named Two-tier architecture. The following figure shows Two-tier architecture:

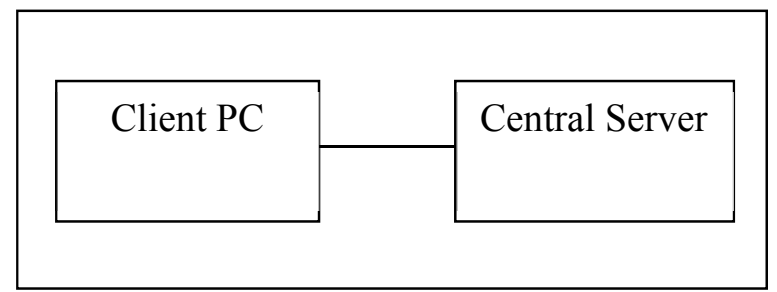

Figure 1: Two-Tier ERP architecture ${ }^{11}$

Before Two-tier appeared, Single-instance ERP systems were used. Single-instance ERP was very expensive and takes too long to arrange organizational change with business models, competitive pressures, needs and global customer demands. Therefore, two-tier ERP made a business solution at that time. It introduced new ERP that was a bit lower in cost, higher in processing, and direct in communication. However, Two-tier ERP had lots of drawback such as performance will decrease as number of clients increase, no database change or modification can be added and limited flexibility in functions movement from server to another. ${ }^{12}$ Two-tier architecture design was used by many vendors such as Epicor, NetSuite, Openbravo, SAP, and Microsoft.

\subsection{Three-Tier architecture}

Three-tier ERP architecture was introduced as a solution for two-tier ERP problems. It consists of three layers such as Presentation layer (Graphical User Interface (GUI)), Application layer, and database layer. Presentation Layer is where data is presented for clients. Application layer is responsible for distributing requests across different applications servers and for business logic execution. ${ }^{13}$ Furthermore, Application layer acts as an interface that facilitate communication between Database layer and User interface (presentation layer). Database layer is responsible of data storage, edit, add, and delete.

Three-tier architecture presents new technologies such as database server and its ability to modify data within server. In addition, Application server distribution among varies applications in also a new advantage added to the architecture design. Three-tier architecture is more scalable, reliable, and flexible. It provides easier implementation to reusable components. On the other hand, Three-tier architecture has a complex and a bit expensive design. Three-tier architecture design was used by SAP vendor named SAP R/2, also Epicor, Oracle, and Microsoft used it.

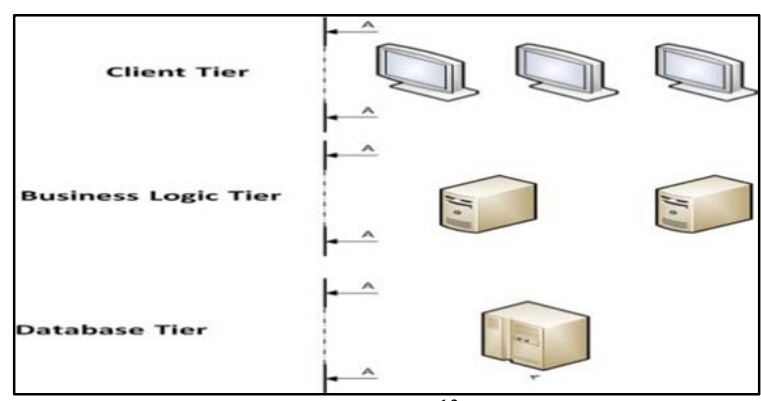

Figure 2: Three-tier architecture ${ }^{13}$

\subsection{Web-based architecture}

With entrance of internet widely in technology world, ERP system developer improved ERP system to coop with internet and web changes. Web based ERP design focused on new technology arises and on migration from Client/server architecture to web-based client/server architecture. It is consists of three layers user interface (web browser), web server, and application/data server. ${ }^{14}$ User interface layer moved from GUI interface to web browser interface. Web server is responsible for HTML, and ASP transactions and act as a gateway between Application/data server and user interface (web browser). Web application/ data server is responsible for application distribution and database storage. The following figure represents web-based architecture:

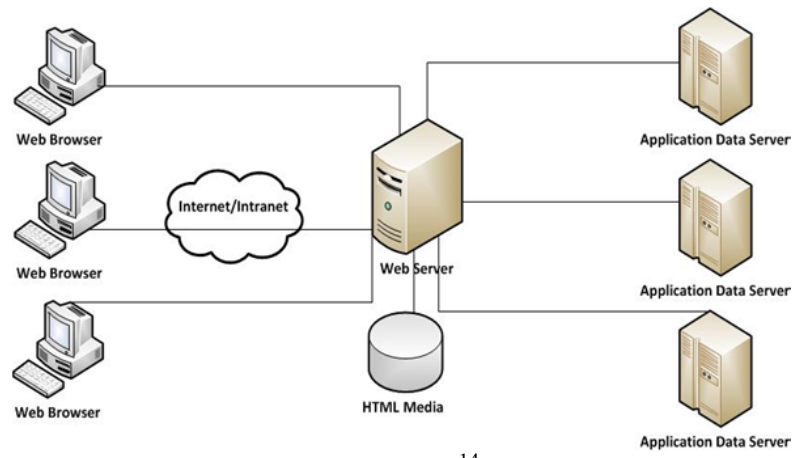

Figure 3: Web-based architecture ${ }^{14}$ 
Web-based architecture has multi-tier client-server architecture as shown in Figure 3. The Web browser is the user interface (client), while the Web server reads and answers the client requests by sending back Web pages. Distant applications and databases can be accessed by the browser through a Web server gateway. Middle-tier communication software layer is perform extracting and sending parameters passed through the HTTP protocol to the remote application and formatting the results into Web pages. ${ }^{15}$

Web-based architecture introduced web-based systems technology that was used for the first time in ERP systems and introduced new Web-based ObjectOriented Model (WOOM). This new technology allows system-to-system integration that facilitates processes between multiple systems. In addition, Web-based architecture reaches higher performance because of web server layer that integrate ERP applications with existing systems and web browser. This architecture design makes faster client/server response, improve data integrity, perform easier modifications, and higher flexibility. ${ }^{16}$ Web-based architecture main drawback is system security and internet security. Web-based architecture design was used by SAP named SAP R3, also used by Microsoft, Oracle, NetSuite, and many other vendors that tried to use and implement their ERP systems based on it.

\section{Technologies and trends affecting ERP development}

\subsection{Web technologies}

Over the old century, ERP begin as a system that only large manufacturing companies can implement. Then ERP moved and spread to smaller companies in all areas and sectors like retail, distribution, healthcare, and government. This is because of web and web technologies that place the foundation stone of ERP development into web ERP. ${ }^{17}$ They enabled ERP to be more dynamic and customer-centric. In addition, many online services were established such as e-commerce and e-business. These web services supported ERP to contribute in web business and move to be web based system.

\subsection{New programming Language}

Throughout ERP development, programming language was the most important part of development and implementation of the system in all ERP history. Programming language is the transformation key of ERP on which the development of it positively affects ERP growth and improvement. ${ }^{18}$ In addition, programming language is the element that made ERP progress from Client/Application to Client/ Server integrated system. Through the enlargement of programming language to web programming such as XML, PHP; ERP implemented new versions that support web services and applications.

\subsection{Web-based System}

Nowadays, internet has affected companies and organizations life on which manufacturing enterprise needed to reshape their working models in order to reach competitive advantage in global manufacturing. Enterprise Resource Planning (ERP) became more critical by adding new internet model. Internet implemented new ERP model which is Web-based Object-Oriented Model (WOOM) which is using internet component and technology as object oriented models for quicker, faster implementation and customization. WOOM made a new generation of ERP called web-based ERP. ${ }^{19}$ In addition, WOOM made ERP system defined in term of objects that is represented under object oriented method based on real world entities such as organizations, events, or individual role.

Web-based ERP system has the capabilities of internet access and real time information that result more efficient and accurate business. Moreover, Webbased ERP provide companies data about supply chain and help facilitate e-commerce transactions. The figure down represents old ERP Three-Tier Design before implementing Web-based ERP:

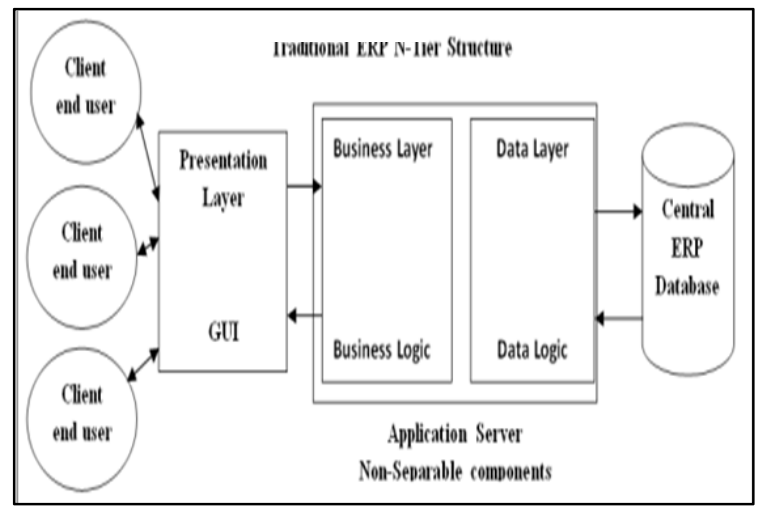

Figure 4: Traditional N-Tier ERP ${ }^{18}$ 
The figure down represents new ERP Design after implementing Web-based ERP:

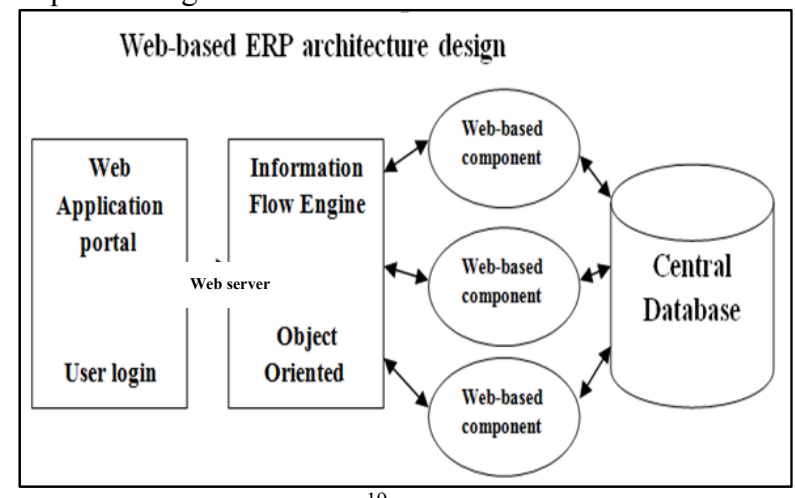

Figure 5: Web-based ERP ${ }^{19}$

\subsection{Mobile Applications}

The increase in mobility users made enterprises figure and recognize the importance of extending their ERP system to mobile applications that provide employees internal system access while mobility. Mobile ERP applications transformed the way businesses engage with customers, partners, and staff by using invented applications that enhance and accelerate the exchange of critical information. ${ }^{20}$ It has many advantages which are: improve quality of service, reach greater productivity, wider business relationship and customer engagement, competitive advantage, and always accessible. Nowadays, most ERP vendors have mobile applications such as SAP ERP system. SAP vendor recently developed mobile business suite application that makes easier access mobility.

\subsection{Cloud Computing}

Cloud computing is a network based service model that enable on demand network access to a shared pool of configurable computing resources such as servers that can rapidly entered. In addition, it is a model that provides special services over the internet; this service could be server, storage, or software. The entrance of this technology made a quick change in ERP systems on which ERP vendors started to implement their ERP systems based on cloud computing models and services. Cloud ERP is hosting an ERP system over the cloud. ${ }^{21}$ This hosting is done through two models IaaS (Infrastructure as a service) and SaaS (Software as a Service). IaaS means buy need Infrastructures over the cloud such as servers. SaaS means its buying or renting services over the cloud such as storage or software's. SaaS is used in cloud ERP on which it takes the responsibility of organizing and managing IT infrastructure (such as servers, operating systems, databases) and processes that are required to run a full solution. ${ }^{22}$ Cloud ERP has many advantages which are less staff, Mobility, easy expandable, cost reduction, and fewer expenses. Business challenges and customer need for cloud technology made Cloud ERP on demand.

\subsection{Security issues}

ERP is becoming a wider solution that's used by small and large organizations and industries. The introduction of ERP into web (web-based ERP), mobile, and cloud made a complex configuration of security issues. This is because of ERP complicated structure that offers security mostly available inside the company. ${ }^{23}$ Furthermore, ERP framework and architecture contains different vulnerability in all the levels from network to application. This means that ERP need to add security modules to all architecture levels in order to reach compatible security.

\subsection{In-memory Computing}

SAP ERP system defines in-memory computing as" a new technology that enables the analysis of very large non-aggregated data at unparalleled speed in local memory". ${ }^{24}$ In-memory computing technology main focus is to store data in primary column format that can compress and store huge amount of information in main memory. Furthermore, this technology facilitate parallel processing in multi core architecture by moving intensive data calculations from the application layer into database layer that end up with faster processing. In-memory technology arise made ERP vendors chose it as a solution that improve their ERP systems. SAP is one of those vendors who invented a new ERP system that support in-memory computing.

\section{Framework of new ERP Design}

As business environment is dramatically changing, companies today face the challenge of increasing competition, expanding market, and raise their customers' expectations. ERP system play an important role in any business environment wither it's a small, 
medium, or large organization. Recently most ERP system developers and vendors are trying to implement and expand their ERP systems. ${ }^{25}$ This is because; new ERP systems implementation will reduce general administrative cost, get better resource utilization, will reduce wastes, reduce decision making time, and reduce operational cost. Furthermore, new ERP system implementation means new or more ERP modules will be added and supported by the system. ERP developers during implementation focus on new system performance and cost reduction also on user friendly interface, secured information, efficient and enhanced technology. These new systems were based on traditional ERP system architecture design such as webbased architecture.

\subsection{New ERP designs:}

Rapid development of information technology supported the movement of ERP to next generation and implementation of new designs. ERP help enterprise systems to change their traditional management methods and offer them more benefits and profits for the enterprise. Most Traditional ERP systems were based on Client/server or browse/server model architecture which is three-tier architecture design. However, the fast development of technology and information systems created six-tier ERP, Cloud ERP, and Mobile ERP which will be explained in the following sections.

\subsection{Six-tier ERP}

As three-tier ERP architecture had lots of deficiencies, six-tier architecture design combines three-tier architecture design and added its own new models. Sixtier architecture consists of six which are: data access layer (DAL), business logic layer (BLL), data service layer (DSL), data mapping layer (DML), components layer (CL), and application layer (AL). The following figure shows six-tier architecture design:

The figure 6 shows that the system is divided into two sections; client and server. Client section contains Application layer and component layer. Each layer has its own function that supports the other layers. Component layer is responsible for data availability for data mapping layer and data handling based on logic relationship between modules. Application Layer contains different components that are provided component layer which support final system result shown to the users. Server section contains four layers that also support each other's. Data access layer (DAL) primarily responsible for original data receive and send, it support business logic layer. Business logic layer (DLL) focuses on domain logic and system implement associated with business need and business process implementation. Data service layer (DSL) main responsibility is to get data from business logic layer or get data from data mapping layer to be processed by business logic layer. In addition, this layer can make decoupling between the data application of the upper levels and the data access and the data handling of the bottom. Data Mapping Layer (DML) main function is to construct communication among the data elements of two different data models. Its final mission is to reach data integration.

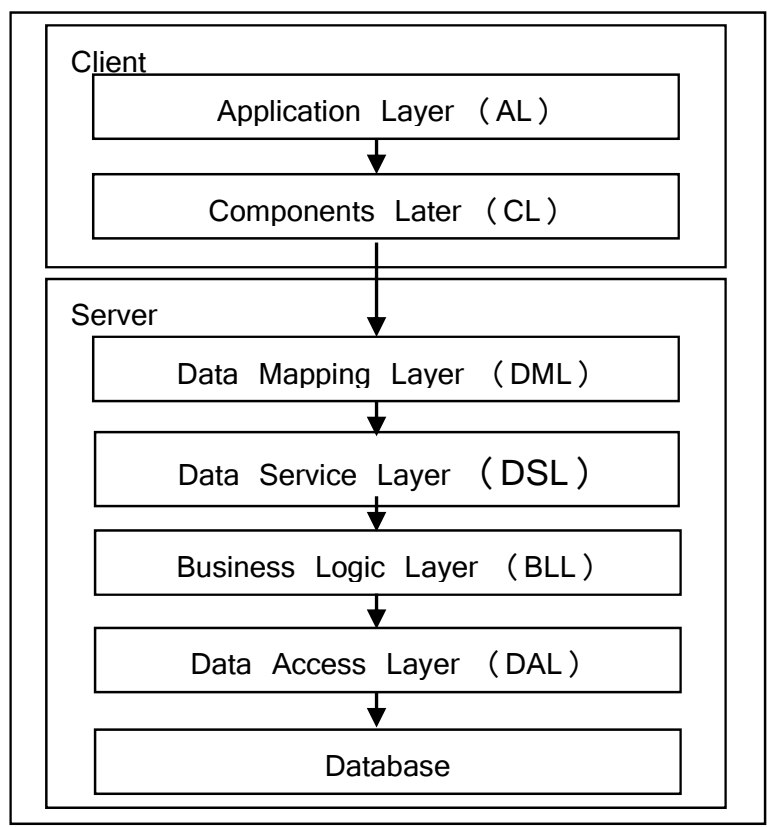

Figure 6: Six-tier architecture ${ }^{26}$

Six-tier architecture showed great solutions to lots of three-tier architecture problems. In six-tier architecture the main focus was to make sure that the clients only see the application and components layer while other layers are transparent to clients and users. Traditional/three-tier system architecture was developed for windows PC system but technology development force systems to migrate from one hardware or software platform to another. ${ }^{26}$ System migration cost used to be very high. In six-tier the architecture of separated 
application layer from the other layers solve migration problems effectively. This will improve platform transplantation and system integration. As a result, this will reduce system development and maintenance cost that's caused by system migration. Six-tier architecture solved one of the biggest problems in three-tier architecture which is providing system decoupling. System decupling reduce maintenance cost and make the system more flexible and make system components less dependable on each other's. Six-tier architecture can make components-based development on which any two or more components later components can form a new application system based on business logic or requirements.

\subsection{Cloud ERP}

ERP system plays an important role in any organizational development. This development will be more reachable when ERP systems are up-to-date with latest technology. Cloud computing entrance made a complete change in enterprises or organizations using ERP systems. ERP moved to cloud ERP because of its improvements, benefits, and flexible system features. Cloud ERP is an approach to enterprise resource planning that use cloud computing platforms and services to make business process transformation more flexible. ${ }^{27}$ In this approach there are three important layers that do cloud services which are Infrastructure as a service (IaaS), Platform as a service (PaaS), Software as a service ( $\mathrm{SaaS})$, and Hardware as a service (HaaS). There are other terms that are used nowadays to name difference resources provided as a service in the cloud such as XaaS. The following figure shows cloud layers:

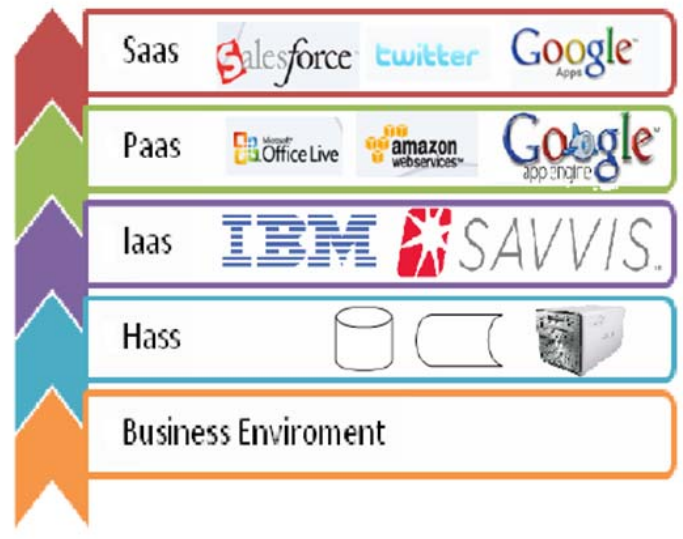

Figure 7: Cloud Layers ${ }^{27}$
Cloud modules layers are modeled to adapt software applications such as ERP systems. Implementing these models in ERP systems will make organizational change from financial and operative perspectives. SaaS module offer low initial cost, low IT resources, low time spent on implementation. It facilitates ERP system work and makes it faster and easier to be developed and implemented. By using SaaS module users or clients will get software service though internet connection without the need to download massive software or infrastructure. ${ }^{28}$ They only need to rent web-based software from a service provider on which it is responsible for the operation.

Cloud ERP system offer customers to use different types of cloud; which are public cloud, private cloud, and hybrid cloud. Public cloud can be accessed by different users where they share common software, hardware, and database. Private cloud can be accessed by specific user and flexible to ass specific features to it. Private cloud usually it is more secure, offer better quality of services, and better data control. Hybrid cloud is a combination of public and private clouds but ondemand. Cloud ERP has many advantages such as lower implementation cost, energy, and maintenance cost. Its elasticity and flexibility is one of the major advantages. ${ }^{29}$ In addition, it offer easy implementation to customer need and features, resource sharing and allocation to different users. Cloud ERP made easy migration to new technologies and software's provided and makes a simpler cloud-based application that can be used remotely by users. Cloud computing has been used by most vendors of ERP system and each vendor implemented their own cloud ERP design. Vendors such as plex system, NetSuite, Epicor, Oracle, Infor, SAP, Microsoft, QAD, IQMS, Sage, and ProcessPro. All of these vendors have implemented their Cloud ERP system depends on their needs and clients or customers need.

\subsection{Mobile ERP}

Mobile computing technology has transformed the way today's businesses are done. It made businesses meet their requirements, strategic advantages, and reach customer satisfaction. In many real life cases quick access to data and information's facilitated organizations or enterprises work and implementation. Mobile computing has become more popular area where most 
ERP developers and vendors moved to use it. The main goal of mobile ERP is to make all ERP functionalities available on various mobile phones and wireless devices. A Mobile ERP major technical requirement is information presentation in multiple formats. This is because mobile and wireless devices are established to support different browsers that support various media formats. ${ }^{20}$ Accordingly, it is necessary to transport the content in different markup languages such as WML, XHTML or HTML. Mobile ERP architecture use XML to store data which facilitates data exchange and transformation to other formats. Mobile ERP architecture is divided into four tiers. Tier one: is ERP system database tier. Tier two: is content access engine, cache storage, and Remote Function Call (RFC) Server tier. This tier is responsible for queries building depend on mobile users request and data retrieve in XML format. RFC server is used to enable begging business functions of an ERP system remotely. Tier three: is content extraction engine. This tier takes charge to presentation logic and determines type of browsers used by user's mobile devices. Tier four: is the user interface tier where it encompasses different mobile devices such as WAP-enabled phones, and PDAs with their particular browser and GUI.

Mobile ERP exceeds the technology boarders, becoming a pattern that provides the enterprise with clear strategic business advantages, real time business ability and in an event driven environment. Mobile device improvements and wide wireless bandwidth opened a new door to traditional efficiency and productivity enhancements, cost reductions, and high user satisfaction.

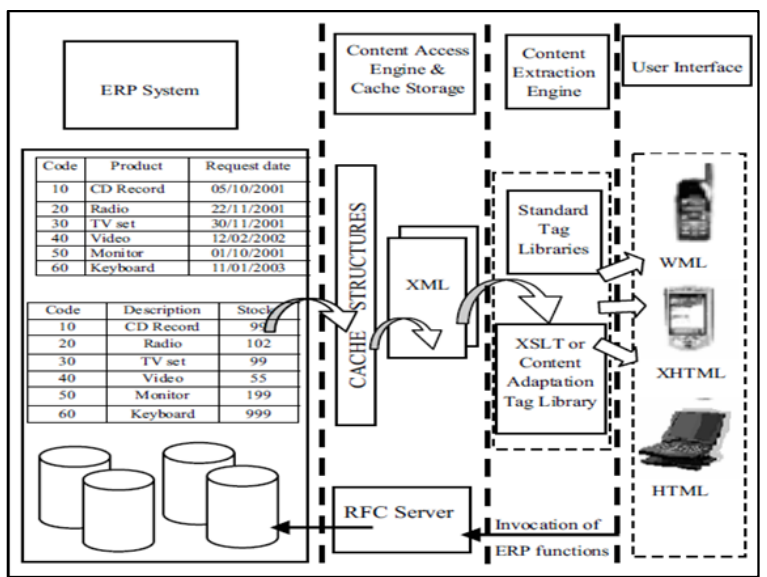

Figure 8: Mobile ERP Architecture ${ }^{20}$
Nowadays, ERP vendors moved to used and develop their ERP system under mobile computing technology. Microsoft, SAP, and Oracle vendors are the most powerful ERP systems that implemented their systems into mobile computing technology. They developed Mobile ERP systems because of its utilized development tools. Furthermore, it makes an easier and quicker to get ERP solutions into the indicators of the mobile workforce. ${ }^{30}$

\section{Discussion}

This paper shows the importance of ERP in providing business solutions for the Enterprises. It indicates how ERP implementation went through many stages over years and how it was used by large vendors. Technology played an important role in ERP development and implementation. It made radical changes in ERP market to be wider and accessible. Every release of new technology, ERP developers coop with it and develop a new system architecture design based on the technology. The major idea of the paper is to specify new ERP architecture designs and vendors that used it. It was shown that Six-tier ERP architecture is the latest ERP architecture design along with could ERP and Mobile ERP systems. ERP architecture designs now are more flexible, on hand, faster, low cost and easier to implement. They provided a great solution to ERP legacy systems architecture. In terms of development, the increasing complexity of business information systems, the need for rapid adaptation to different devices and integration of business concepts are all good reasons of ERP development. As a result, ERP has grown to cover all core business functions for both private and public sector. Moreover, ERP turned to be more modular and moved to be application that's accessed remotely.

\section{Conclusion}

This paper discussed the evolutionary design, trends and deployment of ERP systems. ERP systems are based on multicore parallel computing that facilitates and smooth ERP functions. Web technologies and web services supported ERP development and migration. ERP through years moved from traditional ERP (MRP systems) to move it to cloud and mobile ERP systems. Technology trends development and vendors adaptations moved ERP to new business world full of success and 
realization. Nowadays, business software companies can push the edge of large scale of online transactions processing that ERP can run smoothly. ERP system now can run millions of transactions per seconds. This study will help the developer of ERP to identify the model that affect the future of ERP systems and open the new market for next generation. Furthermore, this study has important implications for service provider, practitioners, researcher, developers and users of ERP. Although our study is an important addition to the literature, we also acknowledge its limitations, mainly the qualitative approach. In the future, it is required to conduct more quantitative research for deeper understanding of users' needs and thoughts.

\section{Conflict of Interest}

There are no known conflicts of interest.

\section{References}

1. Czinkota, M., \& Ronkainen, I. (2012). International marketing. Cengage Learning.

2. Klaus, H., Rosemann, M., \& Gable, G. G. (2000). What is ERP?. Information systems frontiers, 2(2), 141-162.

3. Madurapperuma, T. S., Galkotuwa, Y. K. D., Gunawardana, G. L. C. A., Ramanayaka, R. A. D. K. M., Walpola, D. R. H., \& Thelijjagoda, S. (2009). ERP Deployment in Manufacturing Industry in Sri Lankan Context.

4. Gibson, N., Holland, C. P., \& Light, B. (1999, January). Enterprise resource planning: a business approach to systems development. In Systems Sciences, 1999. HICSS-32. Proceedings of the 32nd Annual Hawaii International Conference on (pp. 9-pp). IEEE.

5. Al-Mashari, M., Al-Mudimigh, A., \& Zairi, M. (2003). Enterprise resource planning: a taxonomy of critical factors. European journal of operational research, 146(2), 352-364. 6. Moller, C. (2005). ERP II: a conceptual framework for next-generation enterprise systems?. Journal of Enterprise Information Management, 18(4), 483-497

7. Krafzig, D., Banke, K., \& Slama, D. (2005). Enterprise SOA: service-oriented architecture best practices. Prentice Hall Professional.

8. Chorafas, D. N. (2001). Integrating ERP, CRM, supply chain management, and smart materials. CRC Press.

9. Addo-Tenkorang, R., \& Helo, P. T. (2014). ERP SaaS value chain: a proposed SaaS model for manufacturing SCM networked activities. International Journal of Business Information Systems, 17(3), 355-372.

10. Jacobson, S., Shepherd, J., D'Aquila, M., \& Carter, K. (2007). The ERP market sizing report, 2006-2011. AMR Research, 29.

11. Wortmann, J. C. (1998). Evolution of ERP systems (pp. 11-23). Springer US.

12. Rajagopal, P. (2002). An innovation-diffusion view of implementation of enterprise resource planning (ERP) systems and development of a research model. Information \& Management, 40(2), 87-114.

13. Kurbel, K., Dabkowski, A., \& Jankowska, A. M. (2003). A multi-tier architecture for mobile enterprise resource planning. In Wirtschaftsinformatik 2003/Band I (pp. 75-93). PhysicaVerlag HD.

14. Tarantilis, C. D., Kiranoudis, C. T., \& Theodorakopoulos, N. D. (2008). A Web-based ERP system for business services and supply chain management: Application to real-world process scheduling. European Journal of Operational Research, 187(3), 1310-1326.

15. Aversano, L., Canfora, G., Cimitile, A., \& De Lucia, A. (2001). Migrating legacy systems to the web: an experience report. In Software Maintenance and Reengineering, 2001. Fifth European Conference on (pp. 148-157). IEEE.

16. Yen, D. C., Chou, D. C., \& Chang, J. (2002). A synergic analysis for web-based enterprise resources planning systems. Computer Standards \& Interfaces, 24(4), 337-346.

17. Ng, J. K., \& Ip, W. H. (2003). Web-ERP: the new generation of enterprise resources planning. Journal of Materials Processing Technology, 138(1), 590-593.

18. Zhang, Z., Lee, M. K., Huang, P., Zhang, L., \& Huang, X. (2005). A framework of ERP systems implementation success in China: An empirical study. International Journal of Production Economics, 98(1), 56-80.

19. Ng, K. C. (2002). The design of an enterprise information system using Hierarchical Design Pyramid and Web-based Object Oriented Model (Doctoral dissertation, The Hong Kong Polytechnic University).

20. Dabkowski, A., \& Jankowska, A. M. (2003, September). Comprehensive framework for mobile ERP system. In Database and Expert Systems Applications, 2003. Proceedings. 14th International Workshop on (pp. 890-894). IEEE.

21. Lenart, A. (2011). ERP in the Cloud-Benefits and Challenges. In Research in systems analysis and design: Models and methods (pp. 39-50). Springer Berlin Heidelberg. 22. Raihana, G. F. H. (2012). Cloud ERP-A Solution Model. International Journal of Computer Science and Information Technology \& Security, 2(1), 76-79.

23. Grabski, S. V., Leech, S. A., \& Schmidt, P. J. (2011). A review of ERP research: A future agenda for accounting information systems. Journal of Information Systems, 25(1), 37-78.

24. Färber, F., May, N., Lehner, W., Große, P., Müller, I., Rauhe, H., \& Dees, J. (2012). The SAP HANA Database--An Architecture Overview. IEEE Data Eng. Bull., 35(1), 28-33.

25. Lee, J., Siau, K., \& Hong, S. (2003). Enterprise Integration with ERP and EAI. Communications of the ACM, 46(2), 5460.

26. Qin, Y., \& Wei, J. (2013, June). The Solution of Enterprise ERP Based on Six-tier Architecture. In Computational and Information Sciences (ICCIS), 2013 Fifth International Conference on (pp. 616-618). IEEE.

27. Nakul, P. (2012). Software package comparison for cloudbased ERP system, retrieved from http://www.slideshare.net/NakulPatel/a-comparison-of-cloudbased-erp-systems.

28. Buyya, R., Yeo, C. S., Venugopal, S., Broberg, J., \& Brandic, I. (2009). Cloud computing and emerging IT platforms: Vision, hype, and reality for delivering computing 
as the 5th utility. Future Generation computer systems, 25(6), 599-616.

29. Saini, S. L., Saini, D. K., Yousif, J. H., \& Khandage, S. V. (2011). Cloud Computing and Enterprise Resource Planning Systems. In Proceedings of the World Congress on Engineering (Vol. 1, pp. 681-684).

30. Clemens, B., Cata, T., \& Hackbarth, G. (2012). Mobile Device Considerations for Supply Chain and ERP Related Systems. IBIMA Publishing, 151480, 16. 